\title{
ON THE VARIETY GENERATED BY THE MONOID OF TRIANGULAR $2 \times 2$ MATRICES OVER \\ A TWO-ELEMENT FIELD
}

\author{
WEN TING ZHANG, JIAN RONG LI and YAN FENG LUO ${ }^{凶}$
}

(Received 5 July 2011)

\begin{abstract}
Let $\mathscr{T}_{n}(F)$ denote the monoid of all upper triangular $n \times n$ matrices over a finite field $F$. It has been shown by Volkov and Goldberg that $\mathscr{T}_{n}(F)$ is nonfinitely based if $|F|>2$ and $n \geq 4$, but the cases when $|F|>2$ and $n=2,3$ or when $|F|=2$ have remained open. In this paper, it is shown that the monoid $\mathscr{T}_{2}(F)$ is finitely based when $|F|=2$, and a finite identity basis for it is given. Moreover, all maximal subvarieties of the variety generated by $\mathscr{T}_{2}(F)$ with $|F|=2$ are determined.
\end{abstract}

2010 Mathematics subject classification: primary 20M07.

Keywords and phrases: finite basis problem, semigroup of triangular matrices, finite field, semigroup variety, subvarieties.

\section{Introduction}

A variety of algebras is a class of algebraic structures of the same signature which is closed under taking of homomorphic images, subalgebras and direct products. By Birkhoff's theorem [1], a variety of algebras is also the class of all algebraic structures of a given signature satisfying a given set of identities.

Let $S$ be a finite algebra and $\operatorname{Id}(S)$ be the set of identities satisfied by $S$. If there is a finite subset $\Gamma$ of $\operatorname{Id}(S)$ such that every identity of $\operatorname{Id}(S)$ can be deduced from $\Gamma$, then $S$ and the variety generated by $S$ are called finitely based. Otherwise they are called nonfinitely based. A variety $V$ is said to be hereditarily finitely based if every subvariety of $V$ is finitely based. A variety $V$ is locally finite if every finitely generated algebra in $V$ is finite. A locally finite variety $V$ is said to be inherently nonfinitely based if each locally finite variety containing $V$ has no finite basis of identities. Certainly, in this case, the variety $V$ itself is also nonfinitely based.

This research was partially supported by the National Natural Science Foundation of China (No. 10971086), the Mathematical Tianyuan Foundation of China (No. 11126186), the Natural Science Foundation of Gansu Province (No. 1107RJZA218), and the Fundamental Research Funds for the Central Universities (No. lzujbky-2012-12).

(C) 2012 Australian Mathematical Publishing Association Inc. 0004-9727/2012 \$16.00 
One of the most famous problems in the study of identities of finite algebras is Tarski's finite basis problem which asks if there is an algorithm to determine when a finite algebra is finitely based. McKenzie [11] proved that this problem is undecidable for general algebras. However, the solution to this problem is unknown when restricted to most natural classes containing both finitely based and nonfinitely based examples. In particular, the finite basis problem for finite semigroups has been extensively studied since the end of the 1960s, and many important and interesting results about it have been obtained (see [5, 6, 8-10, 12, 14, 16] and references therein); furthermore, there is a very large volume of work concerning this problem within the variety of semigroups $[13,14]$.

Let $\mathscr{T}_{n}(F)$ be the monoid of all upper triangular $n \times n$ matrices over a finite field $F$. It is shown by Volkov and Goldberg [15] that the monoid $\mathscr{T}_{n}(F)$ is inherently nonfinitely based if $|F|>2$ and $n \geq 4$, but the cases where $|F|>2$ and $n=2,3$ and where $|F|=2$ are left open; it is also shown that the monoid $\mathscr{T}_{n}(F)$ is not inherently nonfinitely based when $n<4$ or $|F|=2$ [15]. In this paper, it is shown that the monoid $\mathscr{T}_{2}(F)$ is finitely based when $|F|=2$, and a finite identity basis for it is given. Moreover, all maximal subvarieties of the variety generated by $\mathscr{T}_{2}(F)$ with $|F|=2$ are determined.

In what follows, $F$ always denotes a two-element field.

Notation and background information are given in Section 2. In Section 3 it is shown that $\mathscr{T}_{2}(F)$ is finitely based by exhibiting an explicit finite basis. All maximal subvarieties of the variety generated by $\mathscr{T}_{2}(F)$ are presented in Section 4.

\section{Preliminaries}

Most of the notation and background material of this paper are given in this section. The reader is referred to $[1,4]$ for any undefined notation and terminology.

Letters and words. We use $\mathcal{X}^{*}$ and $\mathcal{X}^{+}$to denote the free monoid and free semigroup on an alphabet $\mathcal{X}$, respectively. Elements of $\mathcal{X}$ are referred to as letters and elements of $\mathcal{X}^{*}$ and $\mathcal{X}^{+}$are referred to as words.

Let $x$ and $y$ be any letters and $\mathbf{w}$ be any word. Then:

- $\quad$ the content of $\mathbf{w}$, denoted by $\mathrm{C}(\mathbf{w})$, is the set of all letters occurring in $\mathbf{w}$;

- $\quad$ the number of occurrences of the letter $x$ in $\mathbf{w}$ is denoted by $\mathrm{m}(x, \mathbf{w})$;

- $\quad x$ is simple in $\mathbf{w}$ if $\mathrm{m}(x, \mathbf{w})=1$, or nonsimple otherwise;

- $\quad$ the set of simple letters of $\mathbf{w}$ is denoted by $\operatorname{sim}(\mathbf{w})$;

- $\quad$ a word $\mathbf{w}$ is simple if each of its letters is simple in it, that is, $\operatorname{sim}(\mathbf{w})=\mathrm{C}(\mathbf{w})$;

- the initial of a word $\mathbf{w}$, denoted by ip(w), is the simple word obtained from $\mathbf{w}$ by retaining the first occurrence of each letter;

- the final of a word $\mathbf{w}$, denoted by $\mathrm{fp}(\mathbf{w})$, is the simple word obtained from $\mathbf{w}$ by retaining the last occurrence of each letter;

- $\quad$ if $x$ precedes the first occurrence of $y$ in the word $\mathbf{w}$, let $\overrightarrow{\mathrm{m}}_{y}(x, \mathbf{w})$ denote the number of occurrences of $x$ before the first $y$ in $\mathbf{w}$; 
- $\quad$ if $x$ succeeds the last occurrence of $y$ in the word $\mathbf{w}$, let $\overleftarrow{\mathrm{m}}_{y}(x, \mathbf{w})$ denote the number of occurrences of $x$ after the last $y$ in $\mathbf{w}$;

- $\quad$ let $\operatorname{mix}(\mathbf{w})$ denote the word obtained from $\mathbf{w}$ by retaining the first and the last occurrences of each letter.

ExAmple 2.1. Suppose that $\mathbf{w}=x^{2} z x y^{2} x t z x^{3} t^{4}$. Then:

(1) $\mathrm{C}(\mathbf{w})=\{x, y, z, t\}$, ip $(\mathbf{w})=x z y t$ and $\mathrm{fp}(\mathbf{w})=y z x t$;

(2) $\mathrm{m}(x, \mathbf{w})=7, \mathrm{~m}(y, \mathbf{w})=\mathrm{m}(z, \mathbf{w})=2$, and $\mathrm{m}(t, \mathbf{w})=5$;

(3) $\overrightarrow{\mathrm{m}}_{y}(x, \mathbf{w})=3, \overleftarrow{\mathrm{m}}_{y}(x, \mathbf{w})=4, \overrightarrow{\mathrm{m}}_{z}(x, \mathbf{w})=2$ and $\overleftarrow{\mathrm{m}}_{z}(x, \mathbf{w})=3$;

(5) $\operatorname{mix}(\mathbf{w})=x z y y t z x t$.

Identities and varieties. An identity is a formal expression $\mathbf{u} \approx \mathbf{v}$ where $\mathbf{u}$ and $\mathbf{v}$ are words. A semigroup $S$ will be said to satisfy $\mathbf{u} \approx \mathbf{v}$ if for every homomorphism $\theta$ from $\mathcal{X}^{+}$into $S, \mathbf{u} \theta=\mathbf{v} \theta$. A semigroup $S$ will be said to satisfy a set of identities $\Sigma$ if it satisfies every identity in $\Sigma$. A set of identities will be said to be satisfied by a semigroup if every identity in the set is satisfied by the semigroup. We denote by $\operatorname{Id}(S)$ the set of all identities in some fixed countably infinite alphabet satisfied by a semigroup $S$.

The variety generated by a semigroup $S$ is denoted by var $S$.

Let $\mathbf{u} \approx \mathbf{v}$ be any identity and $x, y$ be any letters. Then:

- $\quad \mathbf{u} \approx \mathbf{v}$ is $n$-balanced at $x$ if $\mathrm{m}(x, \mathbf{u}) \equiv \mathrm{m}(x, \mathbf{v})(\bmod n)$;

- $\quad \mathbf{u} \approx \mathbf{v}$ is $n$-balanced if it is $n$-balanced at every letter;

- $\quad \mathbf{u} \approx \mathbf{v}$ is $n$-initial-balanced at $x$ if $\mathrm{ip}(\mathbf{u})=\operatorname{ip}(\mathbf{v})$ and $\overrightarrow{\mathrm{m}}_{y}(x, \mathbf{u}) \equiv \overrightarrow{\mathrm{m}}_{y}(x, \mathbf{v})(\bmod n)$ for all $y \in \mathcal{X}$;

- $\quad \mathbf{u} \approx \mathbf{v}$ is $n$-initial-balanced if it is $n$-initial-balanced at every letter;

- $\quad \mathbf{u} \approx \mathbf{v}$ is $n$-final-balanced at $x$ if $\mathrm{fp}(\mathbf{u})=\mathrm{fp}(\mathbf{v})$ and $\overleftarrow{\mathrm{m}}_{y}(x, \mathbf{u}) \equiv \overleftarrow{\mathrm{m}}_{y}(x, \mathbf{v})(\bmod n)$ for all $y \in \mathcal{X}$

- $\quad \mathbf{u} \approx \mathbf{v}$ is $n$-final-balanced if it is $n$-final-balanced at every letter.

Let $\Pi$ be any set of identities. The deducibility of an identity $\mathbf{u} \approx \mathbf{v}$ from $\Pi$ is indicated by $\Pi \vdash \mathbf{u} \approx \mathbf{v}$ or $\mathbf{u} \approx \mathbf{m}$. The variety defined by $\Pi$ is the class of all semigroups that satisfy all identities in $\Pi$; in this case, $\Pi$ is said to be a basis for the variety. The subvariety of var $\mathscr{T}_{2}(F)$ defined by $\Pi$ is denoted by $\mathscr{T} \Pi$.

For any $\mathbf{w} \in \mathcal{X}^{+}$and any $\mathcal{Y} \subset \mathcal{X}$, denote by $\mathbf{w} \mathcal{Y}$ the word obtained from $\mathbf{w}$ by retaining the letters from $\mathcal{Y}$. For any identity $\mathbf{w} \approx \mathbf{w}^{\prime}$, denote by $\mathbf{w} \stackrel{*}{\approx} \mathbf{w}^{\prime}$ the identity system that consists of the identity $\mathbf{w} \approx \mathbf{w}^{\prime}$ and all nontrivial identities of the form $\mathbf{w} \mathcal{Y} \approx \mathbf{w}^{\prime} \mathcal{Y}$ with $\mathcal{Y} \subset \mathcal{X}$. For example, the system $x y x z x \stackrel{*}{\approx} x y z x$ is

$$
\left\{x y x z x \approx x y z x, x y x^{2} \approx x y x, x^{2} z x \approx x z x, x^{3} \approx x^{2}\right\} .
$$

Note that if the identity $\mathbf{w} \approx \mathbf{w}^{\prime}$ is satisfied by a monoid $M$, then any identity of the form $\mathbf{w} \mathcal{Y} \approx \mathrm{w}^{\prime} \mathcal{Y}$ is also satisfied by $M$.

Identities of some small semigroups. For any semigroup $S$, let $S^{1}$ be the monoid obtained from $S$ by adjoining an identity element. Denote by $L_{2}, R_{2}, Y_{2}$ and $N_{2}$ the 
left-zero semigroup of order two, the right-zero semigroup of order two, the semilattice of order two and the null semigroup of order two, respectively:

\begin{tabular}{c|ccc|ccc|ccc|cc}
$L_{2}$ & $a$ & $b$ & $R_{2}$ & $a$ & $b$ & $Y_{2}$ & $a$ & $b$ & $N_{2}$ & $a$ & $b$ \\
\hline$a$ & $a$ & $a$ & $a$ & $a$ & $b$ & $a$ & $a$ & $b$ & $a$ & $a$ & $a$ \\
$b$ & $b$ & $b$ & $b$ & $a$ & $b$ & $b$ & $b$ & $b$ & $b$ & $a$ & $a$
\end{tabular}

For any $x \in\{C$, ip, fp $\}$, an identity $\mathbf{u} \approx \mathbf{v}$ is said to be $x$-compliant if $x(\mathbf{u})=x(\mathbf{v})$. The following lemma is well known.

LEMMA 2.2.

(1) An identity is satisfied by the monoid $L_{2}^{1}$ if and only if it is ip-compliant.

(2) An identity is satisfied by the monoid $R_{2}^{1}$ if and only if it is fp-compliant.

(3) An identity is satisfied by the semilattice $Y_{2}$ if and only if it is C-compliant.

(4) An identity $\mathbf{u} \approx \mathbf{v}$ is satisfied by the monoid $N_{2}^{1}$ if and only if $\mathrm{C}(\mathbf{u})=\mathrm{C}(\mathbf{v})$ and $\operatorname{sim}(\mathbf{u})=\operatorname{sim}(\mathbf{v})$.

The following semigroup $A$ (denoted by $S(6,25)$ in [3]) and its dual semigroup $A^{*}$ will be used in this paper:

\begin{tabular}{c|ccccc|cccc}
$A$ & $a$ & $b$ & $c$ & $d$ & $A^{*}$ & $a$ & $b$ & $c$ & $d$ \\
\hline$a$ & $a$ & $a$ & $a$ & $a$ & $a$ & $a$ & $a$ & $c$ & $c$ \\
$b$ & $a$ & $b$ & $c$ & $d$ & $b$ & $a$ & $b$ & $c$ & $d$ \\
$c$ & $c$ & $c$ & $c$ & $c$ & $c$ & $a$ & $c$ & $c$ & $a$ \\
$d$ & $c$ & $d$ & $a$ & $b$ & $d$ & $a$ & $d$ & $c$ & $b$
\end{tabular}

LeMma 2.3. If $\mathbf{u} \approx \mathbf{v}$ is satisfied by the semigroup A, then it is 2-balanced and 2-initialbalanced.

Proof. Note that the semigroup $M_{31}$ in [2] is just the semigroup obtained from $A$ by adjoining a zero element. Then the lemma follows from [2, Lemma 4.5].

By the dual result of Lemma 2.3, we have the following lemma.

LEMMA 2.4. If $\mathbf{u} \approx \mathbf{v}$ is satisfied by the semigroup $A^{*}$, then it is 2-balanced and 2-finalbalanced.

\section{3. $\mathscr{T}_{2}(F)$ is finitely based}

In this section we show that the monoid $\mathscr{T}_{2}(F)$ is finitely based by exhibiting an explicit finite basis.

Clearly, the monoid $\mathscr{T}_{2}(F)$ has eight elements:

$$
\begin{array}{ll}
a=\left(\begin{array}{ll}
0 & 0 \\
0 & 0
\end{array}\right), & b=\left(\begin{array}{ll}
1 & 0 \\
0 & 1
\end{array}\right), \quad c=\left(\begin{array}{ll}
1 & 0 \\
0 & 0
\end{array}\right), \quad d=\left(\begin{array}{ll}
0 & 1 \\
0 & 0
\end{array}\right), \\
e=\left(\begin{array}{ll}
0 & 0 \\
0 & 1
\end{array}\right), \quad f=\left(\begin{array}{ll}
1 & 1 \\
0 & 0
\end{array}\right), \quad g=\left(\begin{array}{ll}
0 & 1 \\
0 & 1
\end{array}\right), \quad h=\left(\begin{array}{ll}
1 & 1 \\
0 & 1
\end{array}\right) .
\end{array}
$$


The multiplication table of $\mathscr{T}_{2}(F)$ is given by

\begin{tabular}{l|llllllll} 
& $a$ & $b$ & $c$ & $d$ & $e$ & $f$ & $g$ & $h$ \\
\hline$a$ & $a$ & $a$ & $a$ & $a$ & $a$ & $a$ & $a$ & $a$ \\
$b$ & $a$ & $b$ & $c$ & $d$ & $e$ & $f$ & $g$ & $h$ \\
$c$ & $a$ & $c$ & $c$ & $d$ & $a$ & $f$ & $d$ & $f$ \\
$d$ & $a$ & $d$ & $a$ & $a$ & $d$ & $a$ & $d$ & $d$ \\
$e$ & $a$ & $e$ & $a$ & $a$ & $e$ & $a$ & $e$ & $e$ \\
$f$ & $a$ & $f$ & $c$ & $d$ & $d$ & $f$ & $a$ & $c$ \\
$g$ & $a$ & $g$ & $a$ & $a$ & $g$ & $a$ & $g$ & $g$ \\
$h$ & $a$ & $h$ & $c$ & $d$ & $g$ & $f$ & $e$ & $b$
\end{tabular}

It is easy to see that $\mathscr{T}_{2}(F)$ is generated by $f, g$ and $h$.

We are ready for our main result in this section.

Theorem 3.1. The monoid $\mathscr{T}_{2}(F)$ is defined by the identities

$$
\begin{aligned}
x h x^{2} t x & \stackrel{*}{\approx} x h t x, \\
x t_{1} y t_{2} x y t_{3} x t_{4} y & \stackrel{*}{\approx} x t_{1} y t_{2} y x t_{3} x t_{4} y, \\
x t_{1} y t_{2} x y t_{3} y t_{4} x & \stackrel{*}{*} x t_{1} y t_{2} y x t_{3} y t_{4} x .
\end{aligned}
$$

It is easy to see that

$$
(3.1 a) \vdash x^{4} \approx x^{2}
$$

The proof of Theorem 3.1 is given at the end of this section. For this purpose, we need to introduce the concept of words in canonical form for the monoid $\mathscr{T}_{2}(F)$.

A word $\mathbf{w}$ with $\mathrm{C}(\mathbf{w})=\left\{x_{1}, \ldots, x_{r}\right\}$ is said to be in canonical form if

$$
\mathbf{w}=a_{1} \mathbf{w}_{1} a_{2} \cdots a_{n} \mathbf{w}_{n} a_{n+1}
$$

where:

(CF1) $\operatorname{mix}(\mathbf{w})=a_{1} a_{2} \cdots a_{n+1}$ with $a_{1}, \ldots, a_{n+1} \in \mathrm{C}(\mathbf{w})$;

$(\mathrm{CF} 2)$ for $i=1, \ldots, n, \mathbf{w}_{i}=x_{1}^{e_{1}} \cdots x_{r}^{e_{r}}$ where $e_{1}, \ldots, e_{r} \in\{0,1\}$.

An identity $\mathbf{u} \approx \mathbf{v}$ is canonical if the words $\mathbf{u}$ and $\mathbf{v}$ are in canonical form.

Lemma 3.2. Let $\mathbf{w}$ be any word. Then there exists some word $\mathbf{w}^{\prime}$ in canonical form such that the identities (3.1) imply the identity $\mathbf{w} \approx \mathbf{w}^{\prime}$.

Proof. It suffices to convert w, using (3.1), into a word in canonical form. Suppose that $\mathrm{C}(\mathbf{w})=\left\{x_{1}, \ldots, x_{r}\right\}$ and $\operatorname{mix}(\mathbf{w})=a_{1} a_{2} \cdots a_{n+1}$ with $a_{1}, \ldots, a_{n+1} \in \mathrm{C}(\mathbf{w})$. Then the word $\mathbf{w}$ can be written in the form

$$
\mathbf{w}=a_{1} \mathbf{w}_{1} a_{2} \cdots a_{n} \mathbf{w}_{n} a_{n+1}
$$

where for each $i$, every letter in $\mathbf{w}_{i}$ is neither a first occurrence nor a last occurrence in $\mathbf{w}$. 
Since the letters of $\mathbf{w}_{i}$ are neither first occurrences nor last occurrences in $\mathbf{w}$, the identities (3.1b) and (3.1c) can be used to permute them within $\mathbf{w}_{i}$ in any manner. In particular, (3.1b) and (3.1c) can be used to permute all letters in $\mathbf{w}_{i}$ so that

$$
\mathbf{w}_{i}=x_{1}^{e_{1}} \cdots x_{m}^{e_{m}}
$$

for some $e_{1}, \ldots, e_{m} \geq 0$; the identities (3.2) and (3.1a) can then be used to reduce the exponents $e_{1}, \ldots, e_{m}$ to numbers in $\{0,1\}$, whence conditions $(\mathrm{CF} 1)$ and $(\mathrm{CF} 2)$ are satisfied.

Lemma 3.3. If $\mathscr{T}_{2}(F)$ satisfies the canonical identity $\mathbf{u} \approx \mathbf{v}$, then $\operatorname{mix}(\mathbf{u})=\operatorname{mix}(\mathbf{v})$.

Proof. Let

$$
\mathbf{u}=a_{1} \mathbf{u}_{1} \cdots a_{m} \mathbf{u}_{m} a_{m+1} \quad \text { and } \quad \mathbf{v}=b_{1} \mathbf{v}_{1} \cdots b_{n} \mathbf{v}_{n} b_{n+1}
$$

be words in canonical form and let $\mathscr{T}_{2}(F)$ satisfy the identity $\mathbf{u} \approx \mathbf{v}$. It suffices to show that $m=n$ and $a_{i}=b_{i}$ for all $i=1, \ldots, n+1$.

Since the subsemigroups $\{b, e, g\},\{b, a, d\}$ and $\{b, c, f\}$ of $\mathscr{T}_{2}(F)$ are isomorphic to $L_{2}^{1}, R_{2}^{1}$ and $N_{2}^{1}$ respectively, it follows from Lemma 2.2 that

$$
\operatorname{ip}(\mathbf{u})=\operatorname{ip}(\mathbf{v}), \quad \mathrm{fp}(\mathbf{u})=\mathrm{fp}(\mathbf{v}), \quad \operatorname{sim}(\mathbf{u})=\operatorname{sim}(\mathbf{v}) \quad \text { and } \quad \mathrm{C}(\mathbf{u})=\mathrm{C}(\mathbf{v}) .
$$

Since the letters $a_{1}, \ldots, a_{m+1}$ and $b_{1}, \ldots, b_{n+1}$ are either first or last occurrences in $\mathbf{u}$ and $\mathbf{v}$ respectively, it follows from the conditions $\mathrm{C}(\mathbf{u})=\mathrm{C}(\mathbf{v})$ and $\operatorname{sim}(\mathbf{u})=\operatorname{sim}(\mathbf{v})$ that $m=n$. Hence it remains to show that $a_{i}=b_{i}$ for all $i$. The condition $\operatorname{ip}(\mathbf{u})=\operatorname{ip}(\mathbf{v})$ clearly implies that $a_{1}=b_{1}$. Suppose that $a_{1} \cdots a_{k-1}=b_{1} \cdots b_{k-1}$. Working toward a contradiction, suppose that $a_{k}=x \neq b_{k}=y$. By symmetry, there are three cases to be considered, depending on whether or not $a_{k}$ and $b_{k}$ are first or last occurrences in $\mathbf{u}$ and $\mathbf{v}$.

Case 1. $a_{k}$ is the first occurrence of the letter $x$ in $\mathbf{u}$ and $b_{k}$ is the first occurrence of the letter $y$ in $\mathbf{v}$. Then the letter $x$ does not occur in $a_{1} \cdots a_{k-1}$ so that $\operatorname{ip}(\mathbf{u})=\operatorname{ip}\left(a_{1} \cdots a_{k-1}\right) x \cdots$. Similarly, $\operatorname{ip}(\mathbf{v})=\operatorname{ip}\left(b_{1} \cdots b_{k-1}\right) y \cdots$. It follows from the assumption $a_{1} \cdots a_{k-1}=b_{1} \cdots b_{k-1}$ that $\operatorname{ip}(\mathbf{u}) \neq \mathrm{ip}(\mathbf{v})$, which is impossible.

Case 2. $a_{k}$ is the last occurrence of the letter $x$ in $\mathbf{u}$ and $b_{k}$ is the last occurrence of the letter $y$ in $\mathbf{v}$. Let $\mathrm{t}\left(a_{1} \cdots a_{k-1}\right)$ be the word obtained from $a_{1} \cdots a_{k-1}$ by retaining the letters which are simple in $a_{1} \cdots a_{n+1}$ and the last occurrence of letters which are nonsimple in $a_{1} \cdots a_{k-1}$. Then $\mathrm{fp}(\mathbf{u})=\mathrm{t}\left(a_{1} \cdots a_{k-1}\right) a_{k} \cdots$. Similarly, $\mathrm{fp}(\mathbf{v})=\mathrm{t}\left(b_{1} \cdots b_{k-1}\right) b_{k} \cdots$. From $\operatorname{sim}(\mathbf{u})=\operatorname{sim}(\mathbf{v})$ and the assumption $a_{1} \cdots a_{k-1}=$ $b_{1} \cdots b_{k-1}$, it follows that $\mathrm{fp}(\mathbf{u}) \neq \mathrm{fp}(\mathbf{v})$, which is impossible.

Case 3. $a_{k}$ is the first occurrence of the letter $x$ in $\mathbf{u}$ and $b_{k}$ is the last occurrence of the letter $y$ in $\mathbf{v}$.

If $x$ is simple in $\mathbf{u}$, then $a_{k}$ is also the last occurrence of the letter $x$ in $\mathbf{u}$ and so $a_{k}=b_{k}$ by Case 2. This gives a contradiction. Similarly, if $y$ is simple in $\mathbf{v}$, then $a_{k}=b_{k}$, which is also a contradiction. Thus we may assume that both $x$ and $y$ are nonsimple and $x \neq y$. 
Since $a_{k}$ is the first occurrence of the letter $x$ in $\mathbf{u}$ and $b_{k}$ is the last occurrence of the letter $y$ in $\mathbf{v}$, we have $x \notin \mathrm{C}\left(a_{1} \mathbf{u}_{1} \cdots a_{k-1} \mathbf{u}_{k-1}\right)$ and $y \in \mathrm{C}\left(b_{1} \mathbf{v}_{1} \cdots b_{k-1} \mathbf{v}_{k-1}\right)$. It follows from the assumption $a_{1} \cdots a_{k-1}=b_{1} \cdots b_{k-1}$ that $y \in \mathrm{C}\left(a_{1} \mathbf{u}_{1} \cdots a_{k-1} \mathbf{u}_{k-1}\right)$ and $x \notin \mathrm{C}\left(b_{1} \mathbf{v}_{1} \cdots b_{k-1} \mathbf{v}_{k-1}\right)$. Hence the last occurrence of $y$ precedes the first occurrence of $x$ in $\mathbf{v}$ by $\mathrm{C}(\mathbf{u})=\mathrm{C}(\mathbf{v})$, and so $\mathbf{v}\{x, y\}=y^{r} x^{s}$ for some integers $r, s \geq 2$. Since $\mathscr{T}_{2}(F)$ satisfies the identity $\mathbf{u}\{x, y\}=\mathbf{v}\{x, y\}$, we have $\operatorname{ip}(\mathbf{u})\{x, y\}=\operatorname{ip}(\mathbf{v})\{x, y\}=y x$ and $\mathrm{fp}(\mathbf{u})\{x, y\}=\operatorname{ip}(\mathbf{v})\{x, y\}=y x$. By the assumption $a_{1} \cdots a_{k-1}=b_{1} \cdots b_{k-1}$ and $b_{k}=y$, it is easy to show that $y$ occurs only once in $a_{1} \cdots a_{k-1}$, and so $y$ occurs in $a_{k+1} \cdots a_{n}$ because $y$ is nonsimple. Hence it follows from $\mathrm{f}(\mathbf{u})\{x, y\}=y x$ that $\mathbf{u}\{x, y\}=y^{t_{1}} x^{t_{2}} y^{t_{3}} \cdots x^{t_{l}}$ for some integers $l \geq 4$ and $t_{i} \geq 1$. Let $\varphi$ denote the substitution into the semigroup $\mathscr{T}_{2}(F)$ :

$$
t \mapsto \begin{cases}e & \text { if } t=x, \\ f & \text { if } t=y .\end{cases}
$$

Then $(\mathbf{v}\{x, y\}) \varphi=f e=d$ and $(\mathbf{u}\{x, y\}) \varphi=f e f \cdots e=a$, which is impossible. Hence $a_{k}=b_{k}$. Therefore $a_{i}=b_{i}$ for all $i=1, \ldots, n+1$ and so $\operatorname{mix}(\mathbf{u})=\operatorname{mix}(\mathbf{v})$.

LEMMA 3.4. The following conditions on a canonical identity $\mathbf{u} \approx \mathbf{v}$ are equivalent:

(a) $\mathscr{T}_{2}(F)$ satisfies the identity $\mathbf{u} \approx \mathbf{v}$;

(b) $\mathbf{u} \approx \mathbf{v}$ is a 2-balanced, 2-initial-balanced and 2-final-balanced identity with $\operatorname{mix}(\mathbf{u})=\operatorname{mix}(\mathbf{v})$

(c) $\mathbf{u} \approx \mathbf{v}$ is trivial.

Proof. (a) implies (b). Let $\mathbf{u} \approx \mathbf{v}$ be a canonical identity and $\mathscr{T}_{2}(F)$ satisfy the identity $\mathbf{u} \approx \mathbf{v}$. Then it follows from Lemma 3.3 that $\operatorname{mix}(\mathbf{u})=\operatorname{mix}(\mathbf{v})$. Since the subsemigroups $\{b, e, g, h\}$ and $\{b, c, f, h\}$ of $\mathscr{T}_{2}(F)$ are isomorphic to semigroups $A$ and $A^{*}$ respectively, it follows from Lemmas 2.3 and 2.4 that $\mathbf{u} \approx \mathbf{v}$ is 2-balanced, 2-initial-balanced and 2-final-balanced.

(b) implies (c). Note that $\mathbf{u} \approx \mathbf{v}$ is a canonical identity with $\operatorname{mix}(\mathbf{u})=\operatorname{mix}(\mathbf{v})$. Let $\operatorname{mix}(\mathbf{u})=\operatorname{mix}(\mathbf{v})=a_{1} a_{2} \cdots a_{n} a_{n+1}$. Then

$$
\mathbf{u}=a_{1} \mathbf{u}_{1} a_{2} \mathbf{u}_{2} \cdots a_{n} \mathbf{u}_{n} a_{n+1} \quad \text { and } \quad \mathbf{v}=a_{1} \mathbf{v}_{1} a_{2} \mathbf{v}_{2} \cdots a_{n} \mathbf{v}_{n} a_{n+1} .
$$

It is shown in the following that $\mathrm{C}\left(\mathbf{u}_{i}\right)=\mathrm{C}\left(\mathbf{v}_{i}\right)$ for all $i=1, \ldots, n$, whence $\mathbf{u}_{i}=\mathbf{v}_{i}$ for each $i$ by the definition of a word in canonical form. Therefore the identity $\mathbf{u} \approx \mathbf{v}$ is trivial.

Suppose, to the contrary, that $\mathrm{C}\left(\mathbf{u}_{k}\right) \neq \mathrm{C}\left(\mathbf{v}_{k}\right)$ for some $k=1, \ldots, n$ and $z \in \mathrm{C}\left(\mathbf{u}_{k}\right) \backslash$ $\mathrm{C}\left(\mathbf{v}_{k}\right)$. It will be convenient to let

$$
\begin{aligned}
\mathbf{u}^{\prime}=a_{1} \mathbf{u}_{1} \cdots a_{k-1} \mathbf{u}_{k-1}, & \mathbf{u}^{\prime \prime}=\mathbf{u}_{k+1} a_{k+2} \cdots \mathbf{u}_{n} a_{n+1}, \\
\mathbf{v}^{\prime}=a_{1} \mathbf{v}_{1} \cdots a_{k-1} \mathbf{v}_{k-1}, & \mathbf{v}^{\prime \prime}=\mathbf{v}_{k+1} a_{k+2} \cdots \mathbf{v}_{n} a_{n+1} .
\end{aligned}
$$

Then it follows from the definition of a word in canonical form that $z \in \mathrm{C}\left(\mathbf{u}^{\prime} a_{k}\right) \cap$ $\mathrm{C}\left(a_{k+1} \mathbf{u}^{\prime \prime}\right)$ in $\mathbf{u}$, and so $z \in \mathrm{C}\left(\mathbf{v}^{\prime} a_{k}\right) \cap \mathrm{C}\left(a_{k+1} \mathbf{v}^{\prime \prime}\right)$ in $\mathbf{v}$ since $\operatorname{mix}(\mathbf{u})=\operatorname{mix}(\mathbf{v})$. There are five cases. 
Case 1. $a_{k}=a_{k+1}=x$. Then $a_{k}$ is the first occurrence of the letter $x$ and $a_{k+1}$ is the last occurrence of the letter $x$ in $\mathbf{u}$ and $\mathbf{v}$. If $z=x$, then $z \notin \mathrm{C}\left(\mathbf{u}^{\prime} \mathbf{u}^{\prime \prime}\right)$ in $\mathbf{u}$ and $z \notin \mathrm{C}\left(\mathbf{v}^{\prime} \mathbf{v}^{\prime \prime}\right)$ in $\mathbf{v}$. It follows that

$$
\mathrm{m}(x, \mathbf{u})=3 \text { and } \mathrm{m}(x, \mathbf{v})=2,
$$

which contradicts the fact that $\mathbf{u} \approx \mathbf{v}$ is 2-balanced. If $z \neq x$, then $z \in \mathrm{C}\left(\mathbf{u}^{\prime}\right) \cap \mathrm{C}\left(\mathbf{u}^{\prime \prime}\right)$ in $\mathbf{u}$ and $z \in \mathrm{C}\left(\mathbf{v}^{\prime}\right) \cap \mathrm{C}\left(\mathbf{v}^{\prime \prime}\right)$ in $\mathbf{v}$. It follows that

$$
\overrightarrow{\mathrm{m}}_{x}(z, \mathbf{u}) \equiv \overrightarrow{\mathrm{m}}_{x}(z, \mathbf{v})(\bmod 2) \quad \text { and } \quad \overleftarrow{\mathrm{m}}_{x}(z, \mathbf{u}) \equiv \overleftarrow{\mathrm{m}}_{x}(z, \mathbf{v})(\bmod 2)
$$

Since

$$
\mathrm{m}(z, \mathbf{u})=\overrightarrow{\mathrm{m}}_{x}(z, \mathbf{u})+1+\overleftarrow{\mathrm{m}}_{x}(z, \mathbf{u}) \quad \text { and } \quad \mathrm{m}(z, \mathbf{v})=\overrightarrow{\mathrm{m}}_{x}(z, \mathbf{u})+\overleftarrow{\mathrm{m}}_{x}(z, \mathbf{u})
$$

it follows that $\mathrm{m}(z, \mathbf{u}) \not \equiv \mathrm{m}(z, \mathbf{v})(\bmod 2)$, a contradiction.

Case 2. $a_{k}=x \neq y=a_{k+1}, a_{k}$ is the first occurrence of the letter $x$ and $a_{k+1}$ is the first occurrence of the letter $y$ in $\mathbf{u}$ and $\mathbf{v}$. Clearly $z \neq y$. If $z=x$, then $z \notin \mathrm{C}\left(\mathbf{u}^{\prime}\right)$ in $\mathbf{u}$ and $z \notin \mathrm{C}\left(\mathbf{v}^{\prime}\right)$ in $\mathbf{v}$. It follows that

$$
\overrightarrow{\mathrm{m}}_{y}(x, \mathbf{u})=2 \text { and } \overrightarrow{\mathrm{m}}_{y}(x, \mathbf{v})=1,
$$

which contradicts the fact that $\mathbf{u} \approx \mathbf{v}$ is 2-initial-balanced. If $z \neq x$, then $z \in \mathrm{C}\left(\mathbf{u}^{\prime}\right) \cap$ $\mathrm{C}\left(\mathbf{u}^{\prime \prime}\right)$ in $\mathbf{u}$ and $z \in \mathrm{C}\left(\mathbf{v}^{\prime}\right) \cap \mathrm{C}\left(\mathbf{v}^{\prime \prime}\right)$ in $\mathbf{v}$. It follows that

$$
\overrightarrow{\mathrm{m}}_{x}(z, \mathbf{u}) \equiv \overrightarrow{\mathrm{m}}_{x}(z, \mathbf{v})(\bmod 2)
$$

Since

$$
\overrightarrow{\mathrm{m}}_{y}(z, \mathbf{u})=\overrightarrow{\mathrm{m}}_{x}(z, \mathbf{u})+1 \quad \text { and } \quad \overrightarrow{\mathrm{m}}_{y}(z, \mathbf{v})=\overrightarrow{\mathrm{m}}_{x}(z, \mathbf{u})
$$

it follows that $\overrightarrow{\mathrm{m}}_{y}(z, \mathbf{u}) \not \equiv \overrightarrow{\mathrm{m}}_{y}(z, \mathbf{v})(\bmod 2)$, a contradiction.

Case 3. $a_{k}=x \neq y=a_{k+1}, a_{k}$ is the first occurrence of the letter $x$ and $a_{k+1}$ is the last occurrence of the letter $y$ in $\mathbf{u}$ and $\mathbf{v}$. If $z \neq x, y$, then $z \in \mathrm{C}\left(\mathbf{u}^{\prime}\right) \cap \mathrm{C}\left(\mathbf{u}^{\prime \prime}\right)$ in $\mathbf{u}$ and $z \in \mathrm{C}\left(\mathbf{v}^{\prime}\right) \cap \mathrm{C}\left(\mathbf{v}^{\prime \prime}\right)$ in $\mathbf{v}$. It follows that

$$
\overrightarrow{\mathrm{m}}_{x}(z, \mathbf{u}) \equiv \overrightarrow{\mathrm{m}}_{x}(z, \mathbf{v})(\bmod 2) \quad \text { and } \quad \overleftarrow{\mathrm{m}}_{y}(z, \mathbf{u}) \equiv \overleftarrow{\mathrm{m}}_{y}(z, \mathbf{v})(\bmod 2)
$$

Since

$$
\mathrm{m}(z, \mathbf{u})=\overrightarrow{\mathrm{m}}_{x}(z, \mathbf{u})+1+\overleftarrow{\mathrm{m}}_{y}(z, \mathbf{u}) \quad \text { and } \quad \mathrm{m}(z, \mathbf{v})=\overrightarrow{\mathrm{m}}_{x}(z, \mathbf{v})+\overleftarrow{\mathrm{m}}_{y}(z, \mathbf{v})
$$

it follows that $\mathrm{m}(z, \mathbf{u}) \not \equiv \mathrm{m}(z, \mathbf{v})(\bmod 2)$, a contradiction. If $z=x$, then $x=z \in \mathrm{C}\left(\mathbf{u}^{\prime \prime}\right) \backslash$ $\mathrm{C}\left(\mathbf{u}^{\prime}\right)$ in $\mathbf{u}$ and $x=z \in \mathrm{C}\left(\mathbf{v}^{\prime \prime}\right) \backslash \mathrm{C}\left(\mathbf{v}^{\prime}\right)$ in $\mathbf{v}$. It follows that

$$
\overleftarrow{\mathrm{m}}_{y}(x, \mathbf{u}) \equiv \overleftarrow{\mathrm{m}}_{y}(x, \mathbf{v})(\bmod 2)
$$

Since

$$
\mathrm{m}(x, \mathbf{u})=2+\overleftarrow{\mathrm{m}}_{y}(x, \mathbf{u}) \quad \text { and } \quad \mathrm{m}(x, \mathbf{v})=1+\overleftarrow{\mathrm{m}}_{y}(x, \mathbf{v})
$$


it follows that $\mathrm{m}(x, \mathbf{u}) \neq \equiv \mathrm{m}(x, \mathbf{v})(\bmod 2)$, a contradiction. If $z=y$, then $y=z \in$ $\mathrm{C}\left(\mathbf{u}^{\prime}\right) \backslash \mathrm{C}\left(\mathbf{u}^{\prime \prime}\right)$ in $\mathbf{u}$ and $y=z \in \mathrm{C}\left(\mathbf{v}^{\prime}\right) \backslash \mathrm{C}\left(\mathbf{v}^{\prime \prime}\right)$ in $\mathbf{v}$. It follows that

$$
\overrightarrow{\mathrm{m}}_{x}(y, \mathbf{u}) \equiv \overrightarrow{\mathrm{m}}_{x}(y, \mathbf{v})(\bmod 2) .
$$

Since

$$
\mathrm{m}(y, \mathbf{u})=\overrightarrow{\mathrm{m}}_{x}(y, \mathbf{u})+2 \text { and } \mathrm{m}(y, \mathbf{v})=\overrightarrow{\mathrm{m}}_{x}(y, \mathbf{v})+1,
$$

it follows that $\mathrm{m}(y, \mathbf{u}) \neq \equiv \mathrm{m}(y, \mathbf{v})(\bmod 2)$, a contradiction.

Case 4. $a_{k}=x \neq y=a_{k+1}, a_{k}$ is the last occurrence of the letter $x$ and $a_{k+1}$ is the first occurrence of the letter $y$ in $\mathbf{u}$ and $\mathbf{v}$. Clearly $z \neq x, y$ and $z \in \mathrm{C}\left(\mathbf{u}^{\prime}\right) \cap \mathrm{C}\left(\mathbf{u}^{\prime \prime}\right)$ in $\mathbf{u}$ and $z \in \mathrm{C}\left(\mathbf{v}^{\prime}\right) \cap \mathrm{C}\left(\mathbf{v}^{\prime \prime}\right)$ in $\mathbf{v}$. It follows that

$$
\overrightarrow{\mathrm{m}}_{y}(z, \mathbf{u}) \equiv \overrightarrow{\mathrm{m}}_{y}(z, \mathbf{v})(\bmod 2) \quad \text { and } \quad \overleftarrow{\mathrm{m}}_{x}(z, \mathbf{u}) \equiv \overleftarrow{\mathrm{m}}_{x}(z, \mathbf{v})(\bmod 2)
$$

Since

$$
\mathrm{m}(z, \mathbf{u})=\overrightarrow{\mathrm{m}}_{y}(z, \mathbf{u})+\overleftarrow{\mathrm{m}}_{x}(z, \mathbf{u})-1 \quad \text { and } \quad \mathrm{m}(z, \mathbf{v})=\overrightarrow{\mathrm{m}}_{y}(z, \mathbf{v})+\overleftarrow{\mathrm{m}}_{x}(z, \mathbf{v})
$$

it follows that $\mathrm{m}(z, \mathbf{u}) \neq \mathrm{m}(z, \mathbf{v})(\bmod 2)$, a contradiction.

Case 5. $a_{k}=x \neq y=a_{k+1}, a_{k}$ is the last occurrence of the letter $x$ and $a_{k+1}$ is the last occurrence of the letter $y$ in $\mathbf{u}$ and $\mathbf{v}$. Clearly $z \neq x$. If $z \neq y$, then $z \in \mathrm{C}\left(\mathbf{u}^{\prime}\right) \cap \mathrm{C}\left(\mathbf{u}^{\prime \prime}\right)$ in $\mathbf{u}$ and $z \in \mathrm{C}\left(\mathbf{v}^{\prime}\right) \cap \mathrm{C}\left(\mathbf{v}^{\prime \prime}\right)$ in $\mathbf{v}$. It follows that

$$
\overleftarrow{\mathrm{m}}_{y}(z, \mathbf{u}) \equiv \overleftarrow{\mathrm{m}}_{y}(z, \mathbf{v})(\bmod 2)
$$

Since

$$
\overleftarrow{\mathrm{m}}_{x}(z, \mathbf{u})=\overleftarrow{\mathrm{m}}_{y}(z, \mathbf{u})+1 \quad \text { and } \quad \overleftarrow{\mathrm{m}}_{x}(z, \mathbf{v})=\overleftarrow{\mathrm{m}}_{y}(z, \mathbf{u})
$$

it follows that $\overleftarrow{\mathrm{m}}_{x}(z, \mathbf{u}) \equiv \overleftarrow{\mathrm{m}}_{x}(z, \mathbf{v})(\bmod 2)$, a contradiction. If $z=y$, then $y=z \in$ $\mathrm{C}\left(\mathbf{u}^{\prime}\right) \backslash \mathrm{C}\left(\mathbf{u}^{\prime \prime}\right)$ in $\mathbf{u}$ and $y=z \in \mathrm{C}\left(\mathbf{v}^{\prime}\right) \backslash \mathrm{C}\left(\mathbf{v}^{\prime \prime}\right)$ in $\mathbf{v}$. It follows that

$$
\overleftarrow{\mathrm{m}}_{x}(y, \mathbf{u})=2 \text { and } \overleftarrow{\mathrm{m}}_{x}(y, \mathbf{v})=1
$$

and so $\overleftarrow{\mathrm{m}}_{x}(y, \mathbf{u}) \equiv \overleftarrow{\mathrm{m}}_{x}(y, \mathbf{v})(\bmod 2)$, a contradiction

Hence $\mathrm{C}\left(\mathbf{u}_{k}\right)=\mathrm{C}\left(\mathbf{v}_{k}\right)$, as required.

(c) implies (a). This is obvious.

Now we are ready to prove the main result of this section.

Proof of Theorem 3.1. With the help of a computer program, one may verify that the monoid $\mathscr{T}_{2}(F)$ satisfies the identities (3.1). It remains to show that any identity $\mathbf{u} \approx \mathbf{v}$ satisfied by the monoid $\mathscr{T}_{2}(F)$ is implied by (3.1). In the presence of Lemma 3.2, it suffices to assume that the identity $\mathbf{u} \approx \mathbf{v}$ is canonical. The identity $\mathbf{u} \approx \mathbf{v}$ is then trivial by Lemma 3.4 and so is vacuously a consequence of the identities (3.1). 
As a direct consequence of Theorem 3.1, we have the following corollary.

Corollary 3.5. The monoid $\mathscr{T}_{2}(F)$ is finitely based.

For any two words $\mathbf{u}$ and $\mathbf{v}$, let $\mathbf{u}^{\prime}, \mathbf{v}^{\prime}$ be their canonical forms respectively. Then the identity $\mathbf{u} \approx \mathbf{v}$ holds in $\mathscr{T}_{2}(F)$ if and only $\mathbf{u}^{\prime}=\mathbf{v}^{\prime}$ by Theorem 3.1 and Lemma 3.4. As the process for reducing a word to canonical form can be completed in polynomial time (see the proof of Lemma 3.2), we have the following proposition.

Proposition 3.6. Let $\mathbf{u}, \mathbf{v}$ be two words. Then whether $\mathbf{u} \approx \mathbf{v}$ holds in $\mathscr{T}_{2}(F)$ or not can be decided in polynomial time.

\section{The maximal subvarieties of $\operatorname{var} \mathscr{T}_{2}(F)$}

In this section, we will determine all maximal subvarieties of the variety generated by the monoid $\mathscr{T}_{2}(F)$. It is shown that var $\mathscr{T}_{2}(F)$ has seven maximal subvarieties, each of which is defined within var $\mathscr{T}_{2}(F)$ by one of the following identities

$$
\begin{gathered}
x^{2} \approx x^{3}, \\
x y x=x y^{3} x, \\
x y^{2} z^{2} y^{2} x \approx x z^{2} y^{2} z^{2} y^{2} x, \\
x y^{2} z^{2} y^{2} x \approx x y^{2} z^{2} y^{2} z^{2} x, \\
x y^{2} z^{2} x \approx x y^{2} z^{2} y^{2} z^{2} x, \\
x y x y x \approx x^{2} y^{2} x, \\
x y x y x \approx x y^{2} x^{2} .
\end{gathered}
$$

For any sets $\Pi_{1}$ and $\Pi_{2}$ of identities, the deduction (3.1) $\cup \Pi_{1} \vdash \Pi_{2}$ is abbreviated to $\Pi_{1} \Vdash \Pi_{2}$.

Let

$$
x y^{2} x \approx x^{2}
$$

Lemma 4.1. Let $\mathbf{u} \approx \mathbf{v}$ be any identity such that $\mathrm{C}(\mathbf{u}) \neq \mathrm{C}(\mathbf{v})$. Then $\mathbf{u} \approx \mathbf{v} \Vdash(4.8)$.

Proof. Suppose that $y \in \mathrm{C}(\mathbf{u}) \backslash \mathrm{C}(\mathbf{v})$ for some $y$. Denote by $\varphi$ the substitution

$$
t \mapsto \begin{cases}x^{2} & \text { if } t \in \mathcal{X} \backslash\{y\}, \\ y^{2} & \text { if } t=y\end{cases}
$$

Then $\mathbf{u} \approx \mathbf{v} \Vdash x y^{2} x \stackrel{(3.1)}{\approx} x(\mathbf{u} \varphi) x \approx x(\mathbf{v} \varphi) x \stackrel{(3.1)}{\approx} x^{2} \vdash(4.8) \Vdash\{(4.3),(4.4),(4.5)\}$.

Lemma 4.2 [10, Theorem 1.1 and Corollary 4.6]. Any variety that satisfies the identities

$$
\begin{gathered}
x^{8} y \approx x^{2} y, \quad x y^{8} \approx x y^{2}, \quad x^{7} y x \approx x y x, \\
x^{2} y x \approx x y x^{2}, \quad x y x z x \approx x^{2} y z x, \quad x y x y \approx x^{2} y^{2}
\end{gathered}
$$

is finitely based. 
Corollary 4.3. Each subvariety of var $\mathscr{T}_{2}(F)$ not containing $Y_{2}$ is finitely based and so $\mathscr{T}\{(4.8)\}$ is hereditarily finitely based.

Proof. It follows from Lemmas 2.2 and 4.1 that the maximal subvariety of $\operatorname{var} \mathscr{T}_{2}(F)$ not containing $Y_{2}$ is $\mathscr{T}\{(4.8)\}$. It is easy to show that $\mathscr{T}\{(4.8)\}$ satisfies all identities in (4.9a). Since

$$
\begin{gathered}
x^{2} y x \stackrel{(4.8)}{\approx} x y^{2} x y x \stackrel{(3.1 c)}{\approx} x y x y^{2} x \stackrel{(4.8)}{\approx} x y x^{2}, \\
x y x z x \stackrel{(3.1 a)}{\approx} x y x^{3} z x \stackrel{(4.10)}{\approx} x^{2} y x^{2} z x \stackrel{(3.1 a)}{\approx} x^{2} y z x, \\
x y x y \stackrel{(3.1 a)}{\approx} x^{3} y x y \stackrel{(4.10)}{\approx} x^{2} y x^{2} y \stackrel{(4.8)}{\approx} x^{2} y^{2},
\end{gathered}
$$

it follows that $\mathscr{T}\{(4.8)\}$ also satisfies all identities in (4.9b). Hence it follows from Lemma 4.2 that each subvariety of $\mathscr{T}\{(4.8)\}$ is finitely based.

Next, we explore the maximal subvarieties of var $\mathscr{T}_{2}(F)$ containing $Y_{2}$, so we always assume that the identity $\mathbf{u} \approx \mathbf{v}$ appearing in what follows is a canonical identity such that $\mathrm{C}(\mathbf{u})=\mathrm{C}(\mathbf{v})$.

Lemma 4.4. Let $\mathbf{u} \approx \mathbf{v}$ be any non-2-balanced identity. Then $\mathbf{u} \approx \mathbf{v} \Vdash(4.1)$.

Proof. Suppose that $\mathbf{u} \approx \mathbf{v}$ is not 2-balanced at the letter $x$, say $\mathrm{m}(x, \mathbf{u}) \equiv 0(\bmod 2)$ and $\mathrm{m}(x, \mathbf{v}) \equiv 1(\bmod 2)$. Denote by $\varphi$ the substitution

$$
t \mapsto \begin{cases}x^{2} & \text { if } t \in X \backslash\{x\} \\ x & \text { if } t=x\end{cases}
$$

Then $\mathbf{u} \approx \mathbf{v} \Vdash x^{2} \stackrel{(3.1)}{\approx} x(\mathbf{u} \varphi) x \approx x(\mathbf{v} \varphi) x \stackrel{(3.1)}{\approx} x^{3} \vdash(4.1)$.

LeMma 4.5. Let $\mathbf{u} \approx \mathbf{v}$ be any 2-balanced identity such that $\operatorname{sim}(\mathbf{u}) \neq \operatorname{sim}(\mathbf{v})$. Then $\mathbf{u} \approx \mathbf{v} \Vdash$ (4.2).

Proof. Suppose that $y \in \operatorname{sim}(\mathbf{u}) \backslash \operatorname{sim}(\mathbf{v})$. Then $\mathrm{m}(y, \mathbf{v})>1$ and $\mathrm{m}(y, \mathbf{v}) \equiv 1(\bmod 2)$ because $\mathbf{u} \approx \mathbf{v}$ is 2-balanced. Denote by $\varphi$ the substitution

$$
t \mapsto \begin{cases}x^{2} & \text { if } t \in \mathcal{X} \backslash\{y\}, \\ y & \text { if } t=y\end{cases}
$$

Then $\mathbf{u} \approx \mathbf{v} \Vdash x y x \stackrel{(3.1)}{\approx} x(\mathbf{u} \varphi) x \approx x(\mathbf{v} \varphi) x \stackrel{(3.1)}{\approx} x y^{3} x \Vdash(4.2)$.

LEMMA 4.6. Let $\mathbf{u} \approx \mathbf{v}$ be any identity.

(i) If $\mathbf{u} \approx \mathbf{v}$ is non-ip-compliant, then $\mathbf{u} \approx \mathbf{v} \Vdash(4.3)$.

(ii) If $\mathbf{u} \approx \mathbf{v}$ is non-fp-compliant, then $\mathbf{u} \approx \mathbf{v} \Vdash(4.4)$.

Proof. It suffices to show that (i) holds. Let $\mathbf{u} \approx \mathbf{v}$ be an identity such that $\mathrm{ip}(\mathbf{u}) \neq \mathrm{ip}(\mathbf{v})$. Then there exist distinct letters $y$ and $z$ such that:

(a) within $\mathbf{u}$, the first occurrence of $y$ precedes the first occurrence of $z$; and

(b) within $\mathbf{v}$, the first occurrence of $z$ precedes the first occurrence of $y$. 
Denote by $\varphi$ the substitution

$$
t \mapsto \begin{cases}x^{2} & \text { if } t \in \mathcal{X} \backslash\{y, z\}, \\ y^{2} z^{2} & \text { if } t=y, \\ z^{2} & \text { if } t=z .\end{cases}
$$

Then $\mathbf{u} \approx \mathbf{v} \Vdash x y^{2} z^{2} y^{2} x \stackrel{(3.1)}{\approx} x(\mathbf{u} \varphi) y^{2} x \approx x(\mathbf{v} \varphi) y^{2} x \stackrel{(3.1)}{\approx} x z^{2} y^{2} z^{2} y^{2} x \vdash$ (4.3).

Lemma 4.7. Let $\mathbf{u} \approx \mathbf{v}$ be any ip-fp-compliant identity $\operatorname{such}$ that $\operatorname{sim}(\mathbf{u})=\operatorname{sim}(\mathbf{v})$ and $\operatorname{mix}(\mathbf{u}) \neq \operatorname{mix}(\mathbf{v})$. Then $\mathbf{u} \approx \mathbf{v} \Vdash(4.5)$.

Proof. Since $\mathbf{u} \approx \mathbf{v}$ is an ip-fp-compliant identity such that $\operatorname{sim}(\mathbf{u})=\operatorname{sim}(\mathbf{v})$ and $\operatorname{mix}(\mathbf{u}) \neq \operatorname{mix}(\mathbf{v})$, there exist distinct letters $y$ and $z \operatorname{such}$ that $\operatorname{mix}(\mathbf{u})\{y, z\} \neq \operatorname{mix}(\mathbf{v})\{y, z\}$.

Note that $\operatorname{sim}(\mathbf{u})=\operatorname{sim}(\mathbf{v})$. If either $y$ or $z$ is simple in $\mathbf{u}$ and $\mathbf{v}$, then $\mathbf{u} \approx \mathbf{v}$ is either non-ip-compliant or non-fp-compliant, contradicting the assumption. Therefore both $y$ and $z$ are nonsimple in $\mathbf{u}$ and $\mathbf{v}$. Assume without loss of generality that

$$
\operatorname{mix}(\mathbf{u})=\cdots y \cdots y \cdots z \cdots z \cdots \quad \text { and } \operatorname{mix}(\mathbf{v})=\cdots y \cdots z \cdots y \cdots z \cdots .
$$

Denote by $\varphi$ the substitution

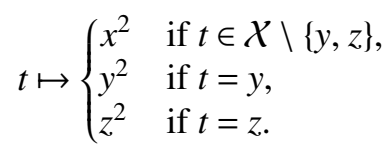

Then $\mathbf{u} \approx \mathbf{v} \Vdash x y^{2} z^{2} x \stackrel{(3.1)}{\approx} x(\mathbf{u} \varphi) x \approx x(\mathbf{v} \varphi) x \stackrel{(3.1)}{\approx} x y^{2} z^{2} y^{2} z^{2} x \vdash(4.5)$.

LEMMA 4.8. Let $\mathbf{u} \approx \mathbf{v}$ be any 2-balanced identity.

(i) If $\overrightarrow{\mathrm{m}}_{y}(x, \mathbf{u}) \not \overrightarrow{\mathrm{m}}_{y}(x, \mathbf{v})(\bmod 2)$ for some $x, y \in \mathrm{C}(\mathbf{u})=\mathrm{C}(\mathbf{v})$, then $\mathbf{u} \approx \mathbf{v} \Vdash$ (4.6).

(ii) If $\overleftarrow{\mathrm{m}}_{y}(x, \mathbf{u}) \not \equiv \overleftarrow{\mathrm{m}}_{y}(x, \mathbf{v})(\bmod 2)$ for some $x, y \in \mathrm{C}(\mathbf{u})=\mathrm{C}(\mathbf{v})$, then $\mathbf{u} \approx \mathbf{v} \Vdash$ (4.7)

Proof. It suffices to show that (i) holds. Without loss of generality, we may assume that

$$
\overrightarrow{\mathrm{m}}_{y}(x, \mathbf{u}) \equiv 0(\bmod 2) \text { and } \overrightarrow{\mathrm{m}}_{y}(x, \mathbf{v}) \equiv 1(\bmod 2) .
$$

Denote by $\varphi$ the substitution

$$
t \mapsto \begin{cases}x^{2} & \text { if } t \in \mathcal{X} \backslash\{x, y\} \\ y^{2} & \text { if } t=y, \\ x & \text { if } t=x\end{cases}
$$

Then it follows from the fact that $\mathbf{u} \approx \mathbf{v}$ is 2-balanced that

$$
\mathbf{u} \approx \mathbf{v} \Vdash x^{2} y^{2} x \stackrel{(3.1)}{\approx}(\mathbf{u} \varphi) x^{l} y^{2} x \approx(\mathbf{v} \varphi) x^{l} y^{2} x \stackrel{(3.1)}{\approx} x y^{2} x y^{2} x \stackrel{(3.1)}{\approx} x y x y x \vdash(4.6),
$$

where $l \equiv \mathrm{m}(x, \mathbf{u}) \equiv \mathrm{m}(x, \mathbf{v})(\bmod 2)$. 
Now we are ready for our main result in this section.

THEOREM 4.9. The varieties

$$
\begin{gathered}
\mathscr{T}\{(4.5)\}=\mathscr{T}\left\{x y^{2} z^{2} x \approx x y^{2} z^{2} y^{2} z^{2} x\right\}, \\
\mathscr{T}\{(4.1)\}=\mathscr{T}\left\{x^{2} \approx x^{3}\right\}, \quad \mathscr{T}\{(4.2)\}=\mathscr{T}\left\{x y x \approx x y^{3} x\right\}, \\
\mathscr{T}\{(4.6)\}=\mathscr{T}\left\{x y x y x \approx x^{2} y^{2} x\right\}, \quad \mathscr{T}\{(4.7)\}=\mathscr{T}\left\{x y x y x \approx x y^{2} x^{2}\right\}, \\
\mathscr{T}\{(4.3)\}=\mathscr{T}\left\{x y^{2} z^{2} y^{2} x \approx x z^{2} y^{2} z^{2} y^{2} x\right\}, \quad \mathscr{T}\{(4.4)\}=\mathscr{T}\left\{x y^{2} z^{2} y^{2} x \approx x y^{2} z^{2} y^{2} z^{2} x\right\}
\end{gathered}
$$

are the only maximal subvarieties of $\operatorname{var} \mathscr{T}_{2}(F)$.

Proof. Since (4.8) $\Vdash\{(4.2)-(4.7)\}$, the maximal subvariety of var $\mathscr{T}_{2}(F)$ not containing $Y_{2}$ is contained in these seven varieties except the second. It is easy to verify that these seven varieties are incomparable. Now the theorem follows immediately from Lemmas 3.4, 4.1, and 4.4-4.8.

Lemma 4.10 [7, Theorem 3.3]. Any variety that satisfies the identities

$$
x^{2} \approx x^{3}, \quad x^{2} y x \approx x y x, \quad x y x^{2} \approx x y x, \quad x y x z x \approx x y z x,
$$

is finitely based.

Corollary 4.11. Each subvariety of $\mathscr{T}\{(4.1)\}$ is finitely based and so $\mathscr{T}\{(4.1)\}$ is hereditarily finitely based.

Proof. It is easy to show that $\mathscr{T}\{(4.1)\}$ satisfies all identities in (4.13). Hence it follows from Lemma 4.10 that each subvariety of $\mathscr{T}\{(4.1)\}$ is finitely based.

The finite basis problem for other subvarieties of var $\mathscr{T}_{2}(F)$ will be considered in a separate paper.

\section{Acknowledgement}

The authors would like to thank the anonymous referees for their valuable comments and suggestions.

\section{References}

[1] S. Burris and H. P. Sankappanavar, A Course in Universal Algebra (Springer, New York, 1981).

[2] C. C. Edmunds, 'On certain finitely based varieties of semigroups', Semigroup Forum 15 (1977), 21-39.

[3] C. C. Edmunds, 'Varieties generated by semigroups of order four', Semigroup Forum 21 (1980), 67-81.

[4] J. M. Howie, Fundamentals of Semigroup Theory (Clarendon Press, Oxford, 1995).

[5] E. W. H. Lee, 'Combinatorial Rees-Sushkevich varieties are finitely based', Int. J. Algebra Comput. 18 (2008), 957-978.

[6] E. W. H. Lee, 'Small semigroups generating varieties with continuum many subvarieties', Order 27 (2010), 83-100.

[7] E. W. H. Lee, 'Finite basis problem for 2-testable monoids', Cent. Eur. J. Math. 9 (2011), 1-22. 
[8] J. R. Li and Y. F. Luo, 'Equational property of certain transformation monoids', Int. J. Algebra Comput. 20(6) (2010), 833-845.

[9] J. R. Li and Y. F. Luo, 'On the finite basis problem for the monoids of triangular boolean matrices', Algebra Universalis 65 (2011), 353-362.

[10] Y. F. Luo and W. T. Zhang, 'On the variety generated by all semigroups of order three', J. Algebra 334 (2011), 1-30.

[11] R. McKenzie, 'Tarski's finite basis problem is undecidable', Int. J. Algebra Comput. 6 (1996), 49-104.

[12] G. Pollák, 'On two classes of hereditarily finitely based semigroup identities', Semigroup Forum 25 (1982), 9-33.

[13] L. N. Shevrin and M. V. Volkov, 'Identities of semigroups', Izv. Vyssh. Uchebn. Zaved. Mat. 11 (1985), 3-47 (in Russian).

[14] M. V. Volkov, 'The finite basis problem for finite semigroups', Sci. Math. Jpn. 53 (2001), 171-199.

[15] M. V. Volkov and I. A. Goldberg, 'Identities of semigroups of triangular matrices over finite fields', Mat. Zametki 73(4) (2003), 502-510 (in Russian); Engl. translation Math. Notes 73(4) (2003) 474-481].

[16] W. T. Zhang and Y. F. Luo, 'The variety generated by a certain transformation monoid', Int. J. Algebra Comput. 18(7) (2008), 1193-1201.

WEN TING ZHANG, Department of Mathematics, Lanzhou University, Lanzhou, Gansu 730000, PR China

JIAN RONG LI, Department of Mathematics, Lanzhou University, Lanzhou, Gansu 730000, PR China

YAN FENG LUO, Department of Mathematics, Lanzhou University, Lanzhou, Gansu 730000, PR China

e-mail: luoyf@lzu.edu.cn 